\title{
Factors associated with progression to severe condition and treatment prolongation in respiratory syncytial virus infection: a retrospective study in Japan
}

\section{Kosuke Oikawa ( $\nabla$ K-oikawa@med.showa-u.ac.jp )}

Showa University https://orcid.org/0000-0002-1439-734X

\section{Manabu Suzuki}

Department of Pediatrics, Showa University School of Medicine

\section{Tomomasa Terada}

Department of Pediatrics, Showa University School of Medicine

\section{Masaya Koganesawa}

Department of Pediatrics, Showa University School of Medicine

\section{Motoichiro Sakurai}

Department of Pediatrics, Showa University School of Medicine

\section{Kazuhiko Matsuhashi}

Department of Pediatrics, Showa University School of Medicine

\section{Hirotaka Ochiai}

Department of Hygiene, Public Health and Preventive Medicine, Showa University School of Medicine

\section{Akatsuki Kokaze}

Department of Hygiene, Public Health and Preventive Medicine, Showa University School of Medicine

Katsumi Mizuno

Johns Hopkins Children's Center

\section{Research article}

Keywords: bronchial asthma, children, lower respiratory tract infection, palivizumab, respiratory syncytial virus

Posted Date: August 20th, 2019

DOI: https://doi.org/10.21203/rs.2.13232/v1

License: (c) (i) This work is licensed under a Creative Commons Attribution 4.0 International License. Read Full License 


\section{Abstract}

Overview Background: In recent years, the epidemic of respiratory syncytial virus (RSV) has been seen in the summer. In daily clinical practice, we noted that RSV infection tends to become more severe and its treatment tends to be longer, particularly in patients hospitalized in the summer. Thus, we investigated factors associated with the progression to severe condition and the prolongation of treatment in RSV infection. Methods: Subjects were pediatric patients diagnosed with RSV infection and hospitalized for treatment during the 5 year s from April 2014 to March 2019. Information on age, sex, season of hospitalization, gestational age, and bronchial asthma of each subject was collected from inpatient medical records. Duration of oxygen administration was used as an index for treatment duration and the use of devices for severe cases as an index for severity. Multiple linear regression analysis and logistic regression analysis were performed to examine the variables associated with duration of oxygen administration and use of a device for severe cases. Results: Data from 298 patients were analyzed. Duration of oxygen administration was significantly associated with bronchial asthma (partial regression coefficient: $0.804, p=0.010$ ). Hospitalization in summer was significantly associated with use of a device for severe condition (adjusted odds ratio: 5.89, 95\% confidence interval: 1.72-20.18). Conclusion: The present study showed that bronchial asthma is a risk factor for prolongation of treatment and infection in summer is a risk factor for progression to severe condition in cases of RSV infection. These findings suggested that children with bronchial asthma and infection in summer need to be treated carefully regardless of their age, sex, and gestational age.

\section{Background}

Respiratory syncytial virus (RSV) is an RNA virus that belongs to the pneumovirus genus, Paramyxoviridae family, and causes respiratory infection in infants [1]. Infection is established and symptoms appear in early infancy when there are maternal antibodies. Half of infants develop symptoms by the age of 1 year, and almost $100 \%$ by the age of 2 . The symptoms become severe in $1 \%$ to $3 \%$ of patients, requiring hospitalization [2]. Only upper respiratory tract infection results from RSV infection in most infants [3]. However, when lower respiratory tract infection including bronchiolitis and pneumonia occurs, expiratory stridor, prolonged expiration, polypnea, and other symptoms are observed and the condition sometimes becomes severe [2]. It is commonly believed that the epidemic occurs in winter in temperate zones, and mainly in December in Japan, while epidemic levels have recently been seen in summer [4]. The same tendency was observed in Tokyo [5].

In our daily clinical practice in recent years, RSV infection has apparently required longer hospitalization and has been more severe in summer than in winter. This tendency was especially prominent in children whose underlying disease was bronchial asthma. Currently, premature infants or children with congenital heart diseases are considered to be at an increased risk for progression of RSV infection to a severe condition and therefore prophylactic palivizumab, an anti-RSV antibody, is indicated for these children in many countries [6]. In response to the recent epidemic of RSV in summer, timing of the administration of palivizumab has been examined in many studies $[7,8]$. However, other risk factors for progression of RSV 
infection to a severe condition have been investigated in only a small number of studies, and it is difficult to predict how hard the treatment would be in daily clinical practice. In this study, we hypothesized that the timing of hospitalization and the presence or absence of bronchial asthma affects the treatment of RSV infection, and retrospectively examined the children whose RSV infection required hospitalization for treatment.

\section{Methods}

The present study subjects were children who were diagnosed with RSV infection and hospitalized for treatment during the 5 years from April 2015 to March 2019 at Showa University Koto Toyosu Hospital (Tokyo, opened at the end of March 2015, a secondary medical care facility) and they were retrospectively examined. RSV infection was diagnosed using a rapid antigen kit, in principle.

Summer was defined as April through September and winter as October through March of the following year, by diving a year into halves using $14.5^{\circ} \mathrm{C}$ as the threshold temperature, referring to the mean temperature by month in the past 5 years in Tokyo [9]. Based on the inpatient medical record, sex, gestational age in weeks, age in months, duration of oxygen administration, presence or absence of bronchial asthma, continuous inhalation of isoproterenol (ISO), and use or no use of high flow nasal cannula (HFNC) were surveyed. Devices for severe cases were defined as continuous inhalation of ISO and use of HFNC. Gestational age in weeks was categorized into the following three groups according to the cutoff ages for administration of palivizumab: $<29$ weeks, 29 to $<35$ weeks, and $\geq 35$ weeks. Duration of oxygen administration was used as an index for treatment duration and use or no use of devices for severe cases as an index for severity. At our hospital, oxygen administration is started when oxygen saturation as measured using pulse oximetry ( $\mathrm{SpO} 2$ ) is decreased to $93 \%$ or lower and the physicians in charge determine the discontinuation timing, considering changes in $\mathrm{SpO} 2$, auscultation findings, systemic condition, and other factors. Presence of bronchial asthma was defined as ongoing treatment in Steps 1 to 4 based on the diagnosis by physicians in charge. Devices for severe cases were introduced when the modified pulmonary index (mPI) [10] was 10 points or higher and the physicians in charge determined that they were necessary. Selection of a device was left to the physicians in charge depending on the circumstances.

In our study, multiple linear regression analysis was conducted to investigate the variables associated with duration of oxygen administration. Logistic regression analysis was performed to evaluate the variables related to use of a device for severe cases. In these models, gestational age, age of the time of hospitalization (months), sex, hospitalization season (summer or winter), and bronchial asthma were included as explanatory variables. $\mathrm{P}<0.05$ was considered statistically significant. JMP® ver 14.0 (SAS) was used for statistical analysis.

\section{Results}


A total of 367 pediatric patients (with overlap, 210 boys and 157 girls) were admitted to this hospital with a diagnosis of RSV infection during the 5 years from April 2015 to March 2019. Among these, 17 subjects were hospitalized more than once ( 15 subjects were hospitalized twice and 1 subject was hospitalized 3 times).

Among 350 patients with no overlap, 8 patients who had an underlying disease of chronic pulmonary disease, cerebral palsy, or chromosomal abnormality, 2 patients between the age of 0 months and 1 month who required ventilator management by tracheal intubation, and 42 patients who had missing data were excluded and finally, 298 patients (175 boys and 123 girls) were analyzed.

Changes in the number of hospitalized patients by month of admission are shown in Figure 1. The number of hospitalized patients was greater in winter than in summer in fiscal year 2014. In contrast, the number of hospitalized patients has been greater in summer than in winter in 2015-2019.

Ages of our study subjects ranged from 0 months to 4 years and 6 months. One hundred and four subjects (34.9\%) were younger than 6 months old, 82 (27.5\%) were aged 6 months or older and younger than 1 year old, 91 (30.5\%) were 1 year old, and 21 (7.0\%) were 2 years or older. Twenty-three subjects (7.7\%) had bronchial asthma and 27 (9.1\%) were treated using devices for severe cases. Palivizumab was indicated for a total of 6 subjects at the time of admission and 3 of them were actually treated with it.

Subject characteristics are shown by hospitalized season (summer or winter) in Table 1. Number of hospitalized patients in summer was greater than in winter. Subjects hospitalized in summer tended to receive the administration of oxygen for a greater number of days and to be treated by the devices for severe cases than those hospitalized in winter.

The factors related to the duration of oxygen administration in patients with RSV infection were examined (Table 2). The presence of bronchial asthma was significantly associated with the duration of oxygen administration (partial regression coefficient; $0.804, p=0.010$ ). Age at the time of hospitalization, sex, hospitalization season and gestational age were not significantly associated with the duration of oxygen administration.

Variables associated with use of device for severe conditions were evaluated (Table 3). Hospitalization season (summer) significantly increased the OR for use of device for severe conditions (OR: $5.98,95 \% \mathrm{Cl}$ : 1.76-20.36). These results remained, even after adjustment for age at the time of hospitalization, sex, gestational age and bronchial asthma.

\section{Discussion}

In this study, we specified, in a dualistic manner, duration of oxygen administration (prolongation of treatment duration) and use or no use of a device for severe cases (severity) as indices for the difficulty of treatment of RSV infection. The results suggested that bronchial asthma is a risk factor for 
prolongation of duration of oxygen administration (prolongation of treatment duration) and morbidity in summer is a risk factor for progression to a severe condition.

Being a premature infant, a history of perinatal respiratory disorder, malnutrition, congenital heart disease, and chronic neurological disorder are known risk factors for progression of RSV infection to a severe condition [11]. American Academy of Pediatrics proposed that bronchiolitis in general involves the risk for progression to a severe condition if it occurs within 12 weeks after birth [12]. For RSV in particular, young age in months has been reported to be a predictive factor for progression to a severe condition [13]. Moreover, RSV infection was reported to be a precipitating factor for bronchial asthma [14] and a risk factor for recurrent wheezing [15]. However, difficulty of the treatment of pediatric patients who have underlying bronchial asthma or who developed RSV infection in summer has been mentioned only in a few reports.

The present study showed that, among pediatric patients with RSV infection, the length of the period during which they required oxygen administration significantly differed between those with bronchial asthma and those without. Considering this finding and the past report suggesting that RSV infection is a precipitating factor for bronchial asthma, we can conclude that the duration of oxygen administration in cases of RSV infection is prolonged when there is underlying bronchial asthma.

The present study also showed that a significantly greater number of patients who were infected with RSV in summer were treated by continuous inhalation of ISO or use of HFNC. Use of ISO or HFNC corresponds to 10 or higher $\mathrm{mPI}$ score and means progression to a severe condition. Patients with bronchial asthma are commonly treated by continuous inhalation of ISO when their condition becomes severe. However, among 23 subjects treated by continuous inhalation of ISO, only 3 had bronchial asthma in our study. Therefore, we speculated that the progression to a severe condition in subjects who had RSV infection in summer does not reflect the worsening of bronchial asthma, but simply suggests the worsening of lower respiratory tract lesions. Although the subjects in this study included many infants younger than 12 months old, age in months at the time of hospitalization was not a factor influencing the progression to a severe condition.

Among the subjects in our study, palivizumab was indicated for a total of 6 subjects at the time of admission and 3 of them were actually treated with it. Devices for severe cases were not used in these 3 subjects treated with palivizumab. The other 3 subjects who did not receive the administration of palivizumab were admitted in September and were infected with RSV before the scheduled day of the administration of palivizumab. Use of a device for severe cases was confirmed in a subject who was not treated with palivizumab. Progression to a severe condition might have been prevented by earlier administration of palivizumab in this subject.

In Japan where the climate is temperate, epidemic of RSV used to be commonly believed to occur in winter, mainly in December. In recent years, however, the epidemic period has changed. In Okinawa, where the climate is subtropical and greatly differs from the climate in other prefectures, the epidemic period has been considered to be from February to September [16]. A similar tendency has recently been 
observed in the Kanto region. A report indicated that RSV was more active at $24^{\circ} \mathrm{C}$ or higher temperature and $6^{\circ} \mathrm{C}$ or lower temperature and most stable when the humidity was $40 \%$ [17]. Another report showed that RSV was more active when the relative humidity was $45 \%$ to $65 \%$ [18]. Still another report demonstrated that RSV preferred a narrow range of humidity and suggested that its detection rate is decreased by $3.8 \%$ when the humidity increases by 1\% [19]. From 2014 to 2019 , the mean temperature in June to September matched the range of temperature at which RSV is considered to become more active. In contrast, relative humidity was higher than the humidity at which RSV is considered to be more active. This suggests that RSV becomes more active at higher relative humidity. It is necessary to continue to observe the epidemics and manifestations carefully, paying attention to climate conditions.

\section{Limitations}

Because this study was a retrospective evaluation and the medical history of the patients was obtained from their medical record, patients with bronchial asthma might not have been completely categorized into the group with this disease. Duration of oxygen administration, continuous inhalation of ISO, and indication of HFNC were not evaluated strictly based on uniform indices such as numbers and scores and therefore might have been influenced by subjective views of primary physician and physicians in charge. Determination of past bronchial asthma cannot necessarily be considered to be in accordance with certain standard diagnostic criteria, either, because presence of bronchial asthma was defined as ongoing treatment from Step 1 to Step 4 based on the diagnosis by physicians in charge. The possibility that these evaluation methods affected the risk factors for prolongation of duration of oxygen administration and progression to a severe condition cannot be denied. Moreover, genetic search for viruses was not performed and it was not determined whether or not any mixed infection with other viruses occurred because of retrospective method of this study. In the future, it is desirable to collect samples and perform these analyses for patients with severe conditions.

\section{Conclusion}

Bronchial asthma was a risk factor for prolongation of treatment duration and infection in summer was a risk factor for progression to a severe condition in RSV infection. The present study suggested that when children with bronchial asthma are infected with RSV, treatment should be provided with consideration of possible long-term administration of oxygen. Moreover, this study indicated that infection in summer needs to be treated carefully. In response to the changes in the epidemic period of RSV in recent years, timing of the administration of palivizumab has been re-examined. However, expansion of the range of age in months for the administration of palivizumab is desired for pediatric patients with bronchial asthma.

\section{Abbreviations}

RSV: respiratory syncytial virus, ISO: isoproterenol, HFNC: high flow nasal cannula mPI: modified pulmonary index 


\section{Declarations}

Acknowledgements

We thank all patients and parents who participated in the study.

\section{Authors' contributions}

$\mathrm{KO}, \mathrm{MS}, \mathrm{TT}, \mathrm{MK}, \mathrm{MS}, \mathrm{MK}$, and KM planned the study. $\mathrm{HO}$ and AK checked the study design and examined the statistical validity. $\mathrm{KO}$ and $\mathrm{HO}$ were in charge of manuscript writing. All authors read and approved the final manuscript.

\section{Funding}

None.

\section{Availability of data and materials}

The datasets used and/or analysed during the current study are available from the corresponding author on reasonable request.

\section{Ethics approval and consent to participate}

This study was reviewed and approved by the institutional review board of Showa University Koto Toyosu Hospital (Institutional Review Board Approval Number, 18T7032).

\section{Consent for publication}

Not applicable.

\section{Competing interests}

The authors declare that they have no competing interests.

\section{References}

1 Tsutsumi H, RS virus(translated). Clinical Microbiology. 2012; 39: 337-42.

2 Tsutsumi H, Yoto Y. RS virus infection(translated). The Journal of Pediatric Practice. 2014; 77: 171-3.

3 Yoshihara S. Asthma onset and RS virus infection(translated). Japanese Journal of Pediatric Pulmonology. 2105; 26: 120-6.

4 Koshiba Y, Tsniguchi H, Itani K, Kaji M, Nakano K, Ota K. Summer Outbreak of Respiratory Syncytial Virus $\triangle R S V \otimes I n f e c t i o n$ in Japan. The Journal of Child Health. 2015; 74: 422-6. 
5 Tokyo Metropolitan Infectious Disease Surveillance Center: Epidemic situation of RS virus infection(translated). http://idsc.tokyo-eiken.go.jp/diseases/rs-virus/rs-virus/. Accessed 23 April 2019.

6 Ginsberg GM, Somekh E, Schlesinger Y. Should we use Palivizumab immunoprophylaxis for infants against respiratory syncytial virus? - a cost-utility analysis. Isr J Health Policy Res. 2018: 17; 63.

7 Shimizu H, Sekine K, Hirai K. Clinical and epidemiological characteristics of 395 patients with respiratory syncytial virus infection from 2008 to 2013 in one pediatric clinic. The Journal of Pediatric Infectious Disease and Immunology. 2015; 27: 119-24.

8 Fujita S, Hirose S, Hamada H, Fujimori M, Yasukawa K, Takanashi J. Epidemic season of respiratory syncytial virus infection is shifting to midsummer in Chiba, Japan: a single center study for clinical characteristics of hospitalized patients in summer, compared with those in sinter. Japanese Society of Emergency Pediatrics. 2019; 18: 16-21.

9 Japan Metrological Agency: Tokyo, Daily Average Temperature Monthly Average $\left({ }^{\circ} \mathrm{C}\right)$.

http://www.data.jma.go.jp/obd/stats/etrn/view/monthly_s3.php?prec_no=44\&block_no=47662. Accessed 3 April 2019.

10 Carroll CL, Sekaran AK, Lerer TJ, et al. A modified pulmonary index score with predictive value for pediatric asthma exacerbations. Ann Allergy Asthma Immunol, 2005: 94; 355-9.

11 Gentile A, Lucion MF, Juarez MDV. Burden of Respiratory Syncytial Virus Disease and Mortality Risk Factors in Argentina: 18 Years Active Surveillance in a Children's Hospital. Pediatr Infect Dis J. 2019; 38: 589-94.

12 American Academy of Pediatrics Subcommittee on Diagnosis and management of bronchiolitis. Diagnosis and management of bronchiolitis. Pediatrics. 2006; 118: 1774-93.

13 Kikuta S, Nishihara M, Sano H, Nabetani M. Prognostic factors of respiratory worsening after admission in otherwise normal patients with respiratory syncytial virus infection. Journal of the Japanese Society of Intensive Care Medicine. 2016; 23: 21-27.

14 Merckx J, Ducharme FM, Martineau C. Respiratory Viruses and Treatment Failure in Children With Asthma Exacerbation. Pediatrics. 2018. doi: 10.1542/peds.2017-4105.

15 Usui $\mathrm{H}$, Odajima Y, Takamura $\mathrm{M}$ et al. CLINICAL EVALUATION OF RECURRENT RESPIRATORY SYNCYTIAL VIRUS INFECTION IN ASTHMATIC CHILDREN. Jpn.J.Pediatr.Allergy Clin.Immunol. 2000; 14: 147-54.

16 Makita T, Genkawa R, Kisato Y, Umeda S, Koh M. The Epidemiology of Respiratory Syncytial Virus Infection in Okinawa Devision of Neonatology $₫$ Okinawa Chubu Hospital. Journal of Japan Society for Premature and Newborn Medicine. 2009; 21: 97-100. 
17 Light M. Respiratory syncytial virus seasonality in southeast Florida: results from three area hospitals caring for children. Pediatr Infect Dis J. 2007: 26; 55-9.

18 Rpbert CW. Temperature Humidity and Ultraviolet B radiation Predict Community Respiratory Syncytial Virus Activity. Pediatr Onfect Dis J 2007: 26: S29-35.

19 Price RHM, Graham C, Ramalingam S. Association between viral seasonality and meteorological factors. Sci Rep. 2019: 9; 92.

\section{Tables}

Due to technical limitations, tables are only available as a download in the supplemental files section.

\section{Figures}

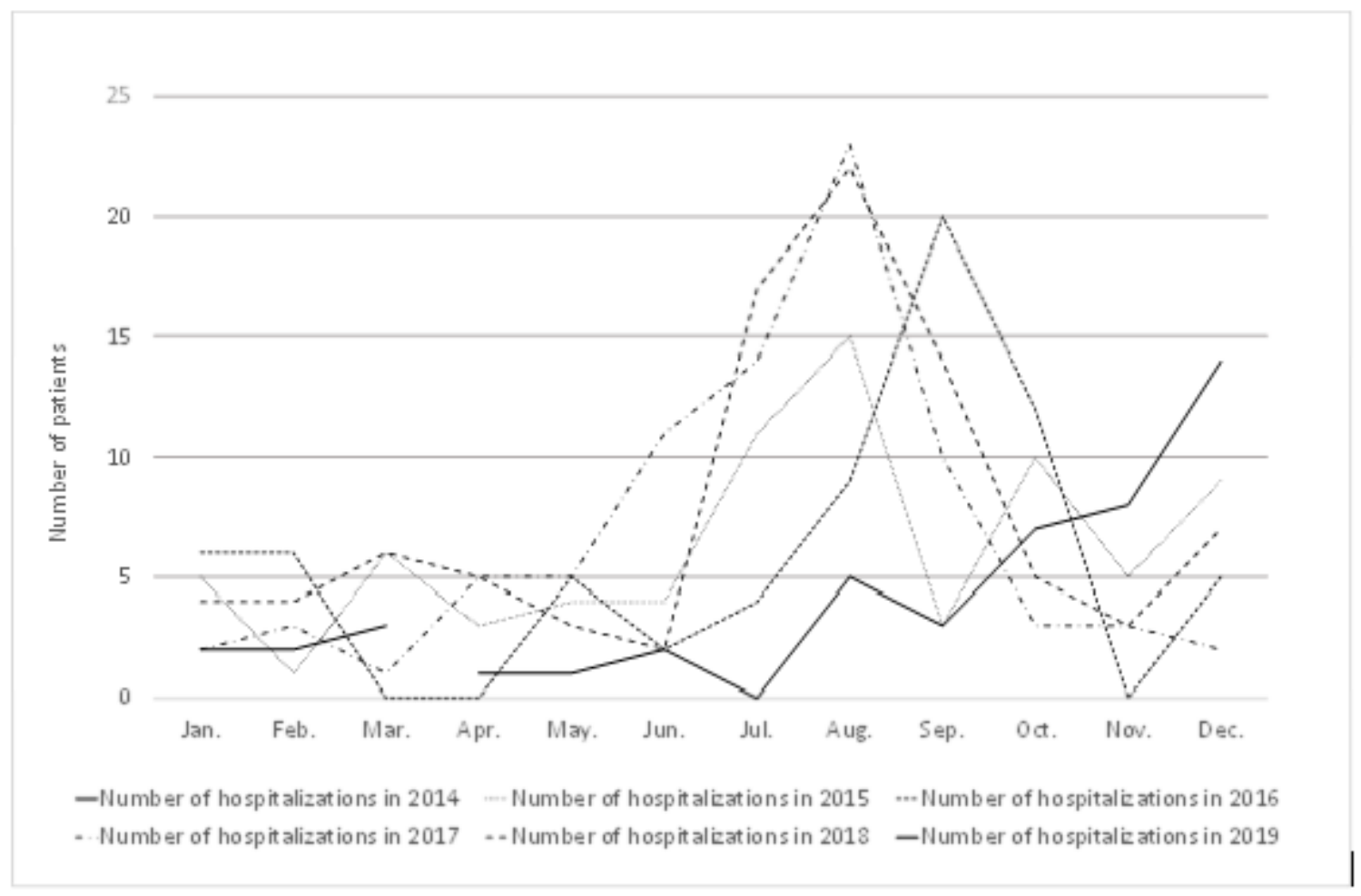

\section{Figure 1}

Number of patients admitted to this hospital for RSV infection by month

\section{Supplementary Files}

This is a list of supplementary files associated with this preprint. Click to download.

- Tables.pdf 